\title{
Implementation of experimental procedures in the PACER system of on-line control
}

\author{
ROGER B. HENRY \\ Department of Psychology, Computer Laboratory \\ University of Nottingham, Nottingham, NG7 $2 R D$ United Kingdom
}

\begin{abstract}
PACER comprises an Executive and associated routines that together provide the run-time facilities commonly needed by experiment control programs. Although the implementation is specific to a particular PDP-11 installation, the nature of the support provided is of more general interest. Simple experimental procedures are straightforwardly handled by direct program control of timing and of external apparatus, and by direct inspection of the states of input lines connected to such apparatus. For more complicated situations, where events may not occur in a strict sequence, it becomes more convenient to make use of priority interrupts to inititiate response to events. Tasks to be performed under interrupt control are coded by the user as special subroutines known as "Actions." It is a feature of PACER that the user can arrange for the Executive to run Actions automatically at specified times. The main program is then able to carry out processing that it would not be able to undertake if it had to make repeated checks on the time.
\end{abstract}

PACER (Program for Assembly language Control of Experiments in Real-time) is a system for PAL-11A programs running on a PDP-11-05 computer with a minimum of $8 \mathrm{~K}$ of core storage. A VT11 display, Laboratory Peripheral System (LPS-11), and extended arithmetic element are also used. Facilities for the implementation of experimental designs have been described in a previous paper (Henry, 1976). The purpose of the present paper is to describe the facilities provided for the implementation of experimental procedures. Users' programs for PACER are run under an Executive that handles all hardware interrupts. From the point of view of the user, PACER is a virtual machine. Communication with the Executive is made via a combination of system variables and subroutine calls. This imposes a structure upon PACER programs that relieves much of the tedium of programming at assembly language level. However, the system can be seen as providing the run-time routines for a high-level control language. Such a system, known as UNCLE (University of Nottinghan Control Language for Experimentation), is under development. The current interest is in the nature of the run-time support provided by PACER as similar solutions could be implemented on other machines.

\section{PRIORITY STRUCTURE}

The PACER priority structure is illustrated in Table 1. This shows the six levels of priority and the relationship between Executive activity and user program activity at each level. The main user program runs at the lowest priority, which is Level 0 . Above this are three levels of priority for tasks that are not carried out by the main program. The tasks are coded by the user as subroutines written in a special format
Table 1

PACER Priority Level Structure

\begin{tabular}{cll}
$\begin{array}{c}\text { Priority } \\
\text { Level }\end{array}$ & $\begin{array}{c}\text { Executive } \\
\text { Activity }\end{array}$ & \multicolumn{1}{c}{$\begin{array}{c}\text { User } \\
\text { Activity }\end{array}$} \\
\hline 5 & $\begin{array}{l}\text { Clock handler, } \\
\text { priority level handler } \\
\text { Device handlers }\end{array}$ & $\begin{array}{l}\text { Device priority } \\
\text { interrupt routines }\end{array}$ \\
4 & & Actions \\
3 & & Main program \\
\hline
\end{tabular}

and are known as "Actions." The priority structure for Actions is implemented as part of the Executive software. Level 4 is implemented by the PDP-11 hardware and is reserved for interrupts from external devices such as the console terminal and response keys. The Executive includes handlers for the interrupts, but, in cases in which fast response is needed, subroutines written by the user may be called at the device priority level. Actions may be called for either immediate or delayed execution from the main program, from device priority interrupt routines, or from any Action. At the highest priority, which is Level 5, a real-time clock generates a hardware interrupt every millisecond. The only noticeable effect of this is that the value of the system variable NOW is incremented. This provides the timebase against which the experiment is paced.

\section{TIMING FACILITIES FOR DIRECT PROGRAM CONTROL}

The system variable NOW is a double-length count of the number of milliseconds that elapse after PACER 
is initially entered. While millisecond accuracy is needed for many applications, at this rate a single 16-bit word overflows after only $65.536 \mathrm{sec}$, and so double-length working is clearly necessary. To time intervals during an experiment, it is necessary to take differences between two values of NOW. Operations upon time values are not only complicated by the requirement for double-length working, but also by the possibility that the carry from the low to the high word will take place during the operation. This possibility arises from the fact that a section of program having control of the machine can be interrupted by a higher priority device or Action after each machine instruction, while many instructions are needed to complete the operation. Subroutines are therefore provided that carry out operations on time values while taking the necessary precautions to insure the correctness of the results. The subroutine calls become instructions in the virtual PACER machine.

The value of NOW at any point in the program may be saved by calling the system routine XSAVE with the result address as a parameter. Subsequently, the difference between a saved value and the current value of NOW is obtained by calling XSINCE with the address of the saved value as a parameter. The result is held in two of the PDP-11 general registers as a number of milliseconds, which is the most convenient format for general working. When it comes to the output of results, which may represent reaction times tc a stimulus, for example, it is more convenient to work in seconds and milliseconds, as these can be separately represented as single-length integers. The routine XM2SEC is therefore provided to convert the doublelength number in the general registers into a number of seconds and milliseconds with the result address given as a parameter.

The three routines XSAVE, XSINCE, and XM2SEC allow the timing of intervals as dependent variables. A slightly different situation arises when a program needs to control the delay between events. It is then convenient to store a (future) value of NOW at which the program should initiate the required operations. This is achieved by calling XTIME with two parameters: the address of a time value to be used as a base and the address at which to store the new time value. The base may be a value stored previously by either XSAVE or NOW itself. The required interval is held in the general registers on entry as a number of milliseconds, and the routine XSEC2M is provided to convert a stored number of seconds and milliseconds into this format.

The program may subsequently use XSINCE to test whether NOW has reached the value stored by XTIME. In fact, XSINCE carries out a double-length test of the sign of the result before returning control to the calling program. This allows conditional branches to be used directly to control the flow of the program.
If it is not yet time to initiate the controlled event, then the program can continue with other processing.

If the program has nothing further to do while waiting for a synchronizing value of NOW, then coding may be simplified by calling another PACER routine. To wait until a given number of milliseconds has elapsed since NOW had a particular value, the routine XWAIT is called with a single parameter pointing to the time value to be used as a base. On return, the pair of general registers used for holding time values will be set to the number of milliseconds by which the program is overdue. As with XSINCE, conditional branches may be used directly to test for lateness. If a program is running late, then explicit tests of the result of XWAIT should be made, allowing for the required degree of tolerance. To wait a given delay from the time that XWAIT is called, it is simply necessary to specify NOW as the base.

\section{INTERRUPT CONTROL OF EVENT TIMING}

As an alternative to direct program control, PACER can arrange for the Executive to run Actions at specified times. This means that the main user program does not have to check whether it is time for a task to be carried out, as this can be done automatically by the Executive. The main program is then able to carry out processing that it would not be able to undertake if it had to make - repeated checks on the time. Examples of tasks that might be carried out in this way are the switching off of a warning tone a given time after it is turned on and the sampling of an analog channel at a given time after the start of a trial.

To $\log$ an Action for later execution, the user program calls the routine XLOG with three parameters: the address of a time value to be used as a base, the address of the Action to be logged, and the address of the head of a waiting line of Actions. The head is XQ3 for priority Level 3, XQ2 for Level 2, or XQ1 for Level 1. As usual, NOW itself can be specified as the base, and the required delay is passed to the routine via the general registers.

An Action is a parameterless subroutine that has three words reserved before the entry point for use by PACER. The first of the words is the address of the Action. XLOG places the time at which the Action is due to be run in the first two words at the Action address. This allows a previously logged Action to be specified as the base for a call of XLOG. (Similarly, an Action address can be given as a parameter to the direct-timing routines.) Actions to be run at the same priority level are arranged in the order of running time, using the third reserved word as a link. When an Action is due, the Executive will run it, provided that is has been assigned a higher priority than the currently running section of program.

All PACER routines are reentrant and may be used 
freely at any priority level within Actions or within the main program, provided only that there are no device clashes and no modifications to common data areas. The values of system variables and working registers used by an interrupt routine must be preserved and restored before return. Special problems are raised by the need to provide a reentrant random number generated for the implementation of experimental designs (Henry, 1976). PACER uses the mixedcongruential method of generating a pseudorandom sequence. The modular arithmetic used in the iteration must not be disturbed by an interrupting program. To allow for repeatability, the sequence retumed must also be independent of events at other priority levels that may occur at unknown times. For these reasons, the generator automatically uses a different seed, multiplier, and constant at each user priority level.

The start of an Action may be delayed by competing Actions at the same or at a higher priority. If timing is critical, then the user program can check for unacceptable delays by calling XSINCE, with the Action address as parameter, on entry to the Action. The Executive itself does not check for lateness, as this would increase overheads and not provide for a degree of tolerance in timing. Clearly, those Actions requiring most precision in timing should be placed in the Level 3 waiting line. The Level 1 waiting line is not normally checked by the Executive to reduce overheads. It can be activated at run-time if needed.

An Action can be logged on again at any time after it has been entered by the Executive. This allows a repetitive Action to be implemented by having the Action $\log$ itself on again before leaving. The base for XLOG should be the Action itself, as using the time at which it was due to be run avoids the accumulation of errors that might result from using NOW as the base. If the Action becomes due again while it is still running, then the priority rules will delay reentry.

Although Actions have no parameters, they can communicate freely with the main program and other Actions using program variables. There is no block structure to restrict the scope of identifiers. A simple example of the use of repetitive Actions is in the periodic sampling of an analog channel. Once sampling is started, it will proceed autonomously of the main program. The Action can count the number of times that it is entered and terminate sampling (by omitting to call XLOG) when the count has reached the value determined by the main program. Sampling rates can also be altered dynamically at run time by making the delay passed to XLOG a program variable.

\section{CALLING ACTIONS FOR UNDELAYED EXECUTION}

To arrange for an Action to be entered as soon as the priority rules allow, the user program calls the routine XACTDO with the Action address and the required priority waiting line address as parameters. If this routine is called from the main program, or from an Action running at a lower priority than that specified for the new Action, then entry will be effected immediately. The Action will have been run before XACTDO has returned control to the calling program. The value of NOW is placed at the Action address before it is run by XACTDO. The first of a series of repetitive calls of an Action is usually started in this way.

If XACTDO is called at the same or at a higher priority than that required for the Action, then the effect will be for the Action to be placed at the head of the appropriate waiting line with the current value of NOW planted at the Action address. It will be entered as soon as it becomes the highest priority Action waiting to be run. This arrangement allows processing to be distributed between priority levels. When an event occurs, operations that must be completed quickly can be run as a high-priority Action. That Action can then $\log$ a lower priority Action to perform any further operations for which timing is not so critical, thereby allowing any competing Actions of higher priority to be entered. This is particularly useful in the case of user subroutines called at the device priority level.

\section{DIGITAL INPUT ROUTINES}

Input and output routines for the direct program control of experimental apparatus present few problems. All that is usually needed is the ability to specify as parameters such variables as the channel or line number, and the operational mode, in a way that is convenient from the point of view of the calling program. The system subroutine takes care of matching these parameters to the format of the status, control, and data registers required by the hardware. Response to digital input lines is somewhat more interesting. These lines form individual binary inputs from devices such as response keys, touch switches, and Schmitt triggers. Changes in the states of the lines may represent events of interest to the experiment control program and therefore need to be detected in some way. Simple situations involving digital inputs are handled by direct program inspection of the states of the lines within program loops. In more complex situations, involving a number of independent groups of input lines and less structure in the sequence of events, it becomes more natural to use interrupts to initiate tasks. This is in accordance with the design philosophy of the PACER system, as illustrated in previous sections.

Response to digital interrupts will usually need to be very fast to allow accurate recording of event times. For this reason, a subroutine can be specified to be run at priority Level 4 when a change of state of interest to the user program occurs within a given group of lines. This is an example of a user routine run at the device priority level (refer to Table 1 ). These routines are not Actions, as they are not linked into a priority 
level waiting line. The distinction is worth maintaining from the user's point of view, as there are some restrictions on the processing that may be performed, arising from the fact that other devices have Level 4 interrupt handlers. In particular, teletype output is buffered via a waiting line that is emptied under interrupt control. Subroutines run at the device priority level must not attempt teletype output, as the Executive will be unable to output characters from the buffer. These routines should, therefore, record any information to be stored and return to the Executive as soon as possible. Any further processing may be undertaken by an Action logged by a call of XACTDO made from the device priority subroutine. The only precaution that is necessary is to insure that the Action will not be logged on again until after it has been entered; this consideration limits the rate of interrupts that can be handled.

PACER currently allows for 16 digital input lines, corresponding to the number of bits in a PDP-11 word. A group of these lines is declared to be of interest to the user by calling the system routine DGON with parameters that point to a pair of words that specify the group and to the corresponding device priority subroutine. The first word of the group specification has bits set for lines to cause an interrupt when going true. The second word has bits set for lines to cause an interrupt when going false. The group includes all lines with at least 1 bit set in the specification words. Any number of groups may be declared, provided that their lines are mutually exclusive. When the subroutine is called, the Executive passes the number of the line causing the interrupt via the general registers, together with information that allows the user to determine the state of the line. Simultaneous events within groups are ignored.

To prevent further interrupts from a group of lines, the routine DGOFF is called with one parameter pointing to the group-specification words used in the call of DGON. Ths user can thus exclude accidental keypresses, for example, made at inappropriate times during the experimental procedure. DGOFF should also be called in a device priority subroutine that logs an Action. The group can then be turned on again within the Action, if necessary.

Sometimes subjects are asked to perform a subsidiary task during an experiment. For example, they may be asked to hold down a response key, but to release it momentarily when a stimulus lamp is turned on. Table 2 uses this example to illustrate how the task can be controlled without the intervention of the main program. The stimulus is turned off when the key is released and turned on again $2 \mathrm{sec}$ later. Response times are output after each trial. Note that if this relatively simple task were to be implemented by direct program inspection, then an explicit test that the subject was pressing the key would have to be included. With interrupt control, this is not necessary. The sequence is started by the main program logging ONNOW with XLOG or XACTDO.
Table 2

Illustrative Use of a Digital Input Routine and Two Actions to Control a Task Without Intervention from the Main Program

\begin{tabular}{|c|c|c|}
\hline Label & Operation & Parameters \\
\hline ONNOW: $0,0,0$ & $\begin{array}{l}\text { turn lamp on } \\
\text { call XSAVE } \\
\text { call DGON } \\
\text { return }\end{array}$ & $\begin{array}{l}\text { RTIM } \\
\text { GROUP 1, KEYREL }\end{array}$ \\
\hline KEYREL: & $\begin{array}{l}\text { turn lamp off } \\
\text { call XSINCE } \\
\text { call DGOFF } \\
\text { call XACTDO } \\
\text { return }\end{array}$ & $\begin{array}{l}\text { RTIM } \\
\text { GROUP } 1 \\
\text { OFFNOW, XQ2 }\end{array}$ \\
\hline OFFNOW: $0,0,0$ & $\begin{array}{l}\text { call XM2SEC } \\
\text { output RTIM } \\
\text { call XSEC2M } \\
\text { call XLOG } \\
\text { return }\end{array}$ & $\begin{array}{l}\text { RTIM } \\
\text { DELAY } \\
\text { OFFNOW, ONNOW, XQ2 }\end{array}$ \\
\hline $\begin{array}{l}\text { RTIM: } 0,0 \\
\text { DELAY: } 0,2 \\
\text { GROUP } 1: 0,1\end{array}$ & & \\
\hline
\end{tabular}

\section{IMPLEMENTATION OF ACTION TIMING}

The algorithm used to implement the software priority levels is shown in simplified form in Table 3. The coding is reentrant to allow Actions that become due to interrupt lower priority Actions that are still running. Termination is guaranteed in all cases by the device of having a dummy waiting line of priority Level 0 . Steps 1 and 18 are implemented directly by the PDP.11 hardware. In the first edition of PACER, the waiting lines of higher priority than the interrupted program were checked after every clock or device priority interrupt to see whether it was time for an Action to be entered. With no Actions logged, the overheads amounted to $13 \%$ of processing time, in other words, $87 \%$ of the time was spent on Level 0. The clock interrupts were provided by the LPS-11 clock, running in repeated intervals of $1 \mathrm{msec}$. In the current version (Edition 3), the variable DELAY has been introduced to improve efficiency. Step 3 is implemented by the LPS-11 clock, running in a single-interval mode in parallel with the PDP-11 CPU. The interrupts that are still necessary for Step 2 are generated by the LPS-1 1 at every count. This has reduced overheads to $3 \%$ when no Actions are running. As a guide to system performance, each time an Action becomes due on priority Level 2 there is an extra overhead of about $450 \mathrm{msec}$. This means, for example, that with an Action running every $10 \mathrm{msec}$ on Level 2, approximately $7.5 \%$ of processing time is spent on Level 5. The remaining $92.5 \%$ is shared by the main program and the Action.

The routine XLOG links the Action to be logged into the specified waiting line so that it precedes all other Actions due to be run at a larger value of NOW. If the waiting line priority is greater than the priority 
Table 3

Algorithm Used to Implement Software Priority Levels

Every Imsec:

1. interrupt current program, pushing priority level of interrupted program onto a stack set system priority to Level 5

2. NOW: $=$ NOW +1

3. if COUNTING $=1$ then DELAY: $=$ DELAY -1 if DELAY $=0$ then COUNTING: $=0$

READY: $=1$

4. if READY $=0$ then go to Step 18 else READY: $=0$

5 . if interrupted program was running at Priority Level 4 then FLAG: $=1$, go to Step 18

6. initialize pointer to waiting line of highest priority XDUE: = maximum value of NOW

7. if priority of Actions on current waiting line is less than or equal to priority of interrupted program then go to Step 18

8. if waiting line is empty then go to Step 16

9. WHEN: = value of NOW at which first Action on waiting line is due

10. if NOW < WHEN then go to Step 15

11. remove Action from waiting line

12. set system priority to waiting line priority

13. run the Action

14. raise system priority to Level 5 , go to Step 8

15. if WHEN < XDUE then XDUE $=$ WHEN, DELAY: $=$ WHEN - NOW, COUNTING: $=1$

16. move pointer to waiting line of next lower priority, go to Step 7

After a device priority interrupt on Level 4

17. if FLAG $=1$ then FLAG: $=0$, set system priority to Level 5, go to Step 6

18. set system priority to priority of interrupted program, popping the stack return control to interrupted program

of the calling program and the Action is due before the current value of XDUE, then the variables XDUE and DELAY are updated and counting is (re)started. XACTDO always places the Action at the head of the waiting line. If the priority rules require immediate entry, then counting is stopped and an interrupt is simulated to Step 6 of Table 3. Whenever an Action becomes due, it is always necessary to start the algorithm by examining the highest priority waiting line, so that counting may be started for any other Action that might need to interrupt it.

\section{APPLICATION}

The application of the PACER routines to the control of experimental procedures is well illustrated by their use in a study of the role of eye movements in the control of hand movements. ${ }^{1}$ In a typical experiment, the subject is required to aim a hand-held probe at a target, starting at rest from a backplate and moving once in a time set by the period of a repetitive tone. The physical backplate, probe, and target are represented on a visual display that provides the only visual feedback of hand position. Touch switches on the backplate and target are connected to digital input lines, and transducers provide analog signals representing horizontal hand position and eye position. Hand position is sampled by a repetitive Action via an analogto-digital converter. A moving average of hand position is used to control the position of a dot on the display that represents the probe. Before a trial is started, the control program must wait for the probe to be held at rest on the backplate. This is arranged by calling the routine DGON to specify a device priority interrupt routine BPSUB to be entered whenever the line from the backplate touch switch changes state. The first time that BPSUB is entered (as indicated by a flag) and the line is true, the Action TONEON is logged using XACTDO. TONEON then sets the output line that controls the tone generator and logs the Action TONEOFF to be entered after a 50-msec delay. TONEOFF clears the output line and relogs TONEON for a delay given by a program parameter. The effect so far is for the repetitive tone to start as soon as the subject touches the backplate with the probe. Meanwhile, if the probe is not held at rest, BPSUB will be entered every time the touch switch changes state. If the line goes true, BPSUB records the time using XSAVE and sets a flag. The flag is cleared if the line goes false. This allows the Action TONEON to test whether the probe is still touching the backplate, and, if so, to find how long it has been there by calling XSINCE. When TONEON finds the probe has been at rest on the backplate for greater than a threshold delay, the trial is started by logging an Action TARGON.

The delay specified for TARGON is a multiple of the tone period. When entered, it checks that the probe is still at rest and then displays the target at a preselected position. The subject is required to aim the probe to hit the target in time with the next sounding of the tone. During the intervening period, eye position is sampled by a repetitive Action via another channel of the analog-to-digital converter. Under some conditions of the experiment, the target or probe may be removed from the display screen while the subject is moving the physical probe to the target. This also is achieved by logging Actions to do the work at a predetermined time after switching on the target. A digital input interrupt routine, entered when the probe hits the physical target, switches the displayed probe and target back on to provide accuracy feedback to the subject.

\section{REFERENCE}

HENRY, R. B. Implementation of experimental design and storage of results in the PACER system of on-line control. Behavior Research Methods \& Instrumentation, 1976, 8. 292-298.

\section{NOTE}

1. The work referred to here was carried out by P. I. Breslaw of the Department of Psychology, Computer Laboratory, University of Nottingham, under a Medical Research Council Grant awarded to Professor C. I. Howarth. 